\title{
Long-acting Injectable Antipsychotics in First-episode Schizophrenia
}

\author{
Hyun-Ghang Jeong ${ }^{1,2}$, Moon-Soo Lee ${ }^{1,2}$ \\ ${ }^{1}$ Department of Psychiatry, Korea University Guro Hospital, ${ }^{2}$ Research Institute of Mental Health, Korea University College of Medicine, \\ Seoul, Korea
}

\begin{abstract}
Antipsychotic medications are important for the successful management of schizophrenia. Continuous treatment with medication is superior in relapse prevention and non-adherence to antipsychotic medication is associated with a poor clinical outcome. Long-acting injectable antipsychotics (LAls) that can guarantee adherence to a treatment regimen could be a useful treatment option. With the introduction of second-generation atypical antipsychotics-long acting injection (SGA-LAI), the risks for extrapyramidal adverse events are decreased. The indications for SGA-LAl have been extended from chronic, stabilized patients to acute psychotic patients. Some studies investigated the use of LAl in first-episode schizophrenia patients and raised the possibility of prescribing LAl as a treatment option. However, there is still limited research using LAl in first-episode schizophrenia. More well-designed, randomized, controlled clinical trials using SGA-LAls in first episode schizophrenia are needed. Additionally, studies on side effects of SGA-LAl in long-term use are required prior to recommending LAl for patients with first episode schizophrenia.
\end{abstract}

KEY WORDS: Antipsychotic agents; Injections; Schizophrenia; Delayed-action preparations.

\section{INTRODUCTION}

Schizophrenia is a chronic disorder characterized by multiple relapses. It is a costly disease as it has an early onset and leads to substantial disability, loss of productivity, and the frequent use of mental health care resources. Frequent relapses are also associated with poor long-term treatment outcomes. Therefore, the prevention of relapses is crucial in the successful management of schizophrenia. Non-adherence to a treatment regimen is commonly reported in schizophrenia patients and is one of the main factors undermining relapse prevention. Relapse is associated with impaired adherence and there are several reasons for non-adherence in schizophrenia patients. Predictors of non-adherence in schizophrenia patients were investigated by Perkins. ${ }^{1)}$ Patients' beliefs about their illness (e.g., impaired insight) and the benefits of treatment, the perceived costs of treatment (e.g., medication adverse events), and barriers to treatment all influence adherences. A positive attitude toward medication ${ }^{2)}$ and having an

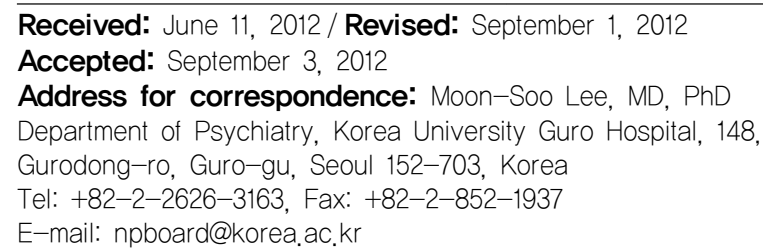

available and supportive family ${ }^{3)}$ were also shown to be related to treatment adherence in schizophrenia patients.

To improve adherence, prescription strategies such as new oral atypical antipsychotics and long-acting injectable antipsychotics (LAI) formulations can be utilized in the pharmacological treatment approach. Moreover, LAIs are convenient for the patient and their family who no longer have the burden and stigma associated with of remembering to take medication on a daily basis. ${ }^{4,5)}$ However, LAIs are not widely used in first-episode schizophrenia although atypical antipsychotic LAI formulations have become available approximate 8 years ago Many clinicians use LAI formulations only for chronic, stabilized schizophrenia patients. This review will summarize the data published at present from studies with a focus on the use of LAI in first episode schizophrenia.

\section{WHY IS THE FIRST EPISODE IMPORTANT?}

It is first necessary to understand the clinical course of schizophrenia. Schizophrenia has several phases: the prodromal, acute, stabilization, and maintenance phases. ${ }^{6}$ The prodromal phase commonly precedes the onset of the formal symptoms of schizophrenia. When the prominent psychotic symptoms exist, a formal diagnosis of first-episode schizophrenia is made. Most patients recover from

(c) This is an Open-Access article distributed under the terms of the Creative Commons Attribution Non-Commercial License (http://creativecommons.org/licenses/by-nc/3.0) which permits unrestricted non-commercial use, distribution, and reproduction in any medium, provided the original work is properly cited. 
the first episode, although many patients continue to have residual symptoms. The first years of the schizophrenia illness have been proposed as a critical period for longterm prognosis. ${ }^{7)}$ In this stage, the antipsychotic treatment response of first-episode schizophrenia patients is better than that of chronic multiple-episode patients. ${ }^{8)}$ Many patients also experience additional episodes. In the subsequent episodes, some patients do not recover to the same level of function as they were able to achieve after the previous episodes. As this relapse pattern continues, the patient experiences a clinical deterioration. The majority of schizophrenia patients experience this clinical deterioration and the degree of deterioration is variable. This deterioration mainly occurs in the early episodes of schizophrenia-during the first 5-10 years after the initial episode. ${ }^{9)}$ After this clinically vulnerable period, the clinical status of patients tends to stabilize except for refractory cases. However, Robinson et al. ${ }^{10)}$ reported that there is also a high relapse rate following the response from the first episode of schizophrenia. They found that five years after the initial recovery in first-episode schizophrenia patients, the cumulative first relapse rate for 104 patients was $81.9 \%$ and the second relapse rate was $78.0 \%$. There was a strong association between antipsychotic drug therapy discontinuation and relapse and antipsychotic medication discontinuation was by far the strongest predictor of relapse in first-episode schizophrenia patients. Many schizophrenia patients hesitate to take antipsychotic medications after recovery from the acute phase. This refusal can be related to the consequent relapses and further functional impairment. For this reason, it is important to manage the early episodes, especially the first episode, in schizophrenia to prevent the clinical deterioration that occurs in the subsequent phase.

\section{PROS AND CONS OF USING LAI FORMULATIONS}

There has been some debate about the effectiveness of maintenance versus intermittent drug treatment in schizophrenia patients. Because the overall exposure to antipsychotic agents can be decreased during the stabilized phase and the incidence of adverse events is decreased, intermittent drug treatment has been used by some researchers. ${ }^{11)}$ However, studies comparing these two treatment regimens show that continuous medication is preferred. An intermittent versus maintenance medication trial was conducted in a 2-year, double-blind, placebo-controlled study. Although not statistically significant, the re- lapse rates were $30 \%$ for the intermittent medication treatment group and $16 \%$ for the maintenance treatment group. Although the intermittent drug treatment group received significantly less medication, there were no significant differences in medication-related adverse events. This study result shows that an intermittent medication strategy has no benefit over a maintenance strategy. ${ }^{12)}$

The two medication strategies were also compared in first-episode schizophrenia patients. In a randomized controlled trial conducted in first-episode schizophrenia patients by the German Research Network on Schizophrenia from November 2000 to May 2004, 23 patients were allocated to the maintenance treatment group and 21 were allocated to the intermittent treatment group. The rates of relapse (19\% versus $0 \%$ ) and deterioration (up to $57 \%$ versus $4 \%$ ) were significantly higher in the intermittent treatment group than in the maintenance treatment group. The results of this study show that maintenance treatment is more effective for relapse prevention, even in first-episode schizophrenia. ${ }^{13)}$

If maintenance treatment is the preferred treatment option, LAI formulations have some advantages over oral medications. However, current clinical practice is not based on these research results. Jaeger and Rossler ${ }^{14)} \mathrm{di}$ rectly investigated the current attitudes, beliefs, and knowledge held by psychiatrists, patients, and relatives concerning depot antipsychotics and compared these attitudes after the introduction of an atypical depot formulation. Patients had more negative attitudes towards depot injections than did psychiatrists and relatives. Patients particularly feared the restriction of autonomy when treated with depot antipsychotics and that the injections might be painful. Approximately $67 \%$ of all patients did not receive information about depot antipsychotics from their psychiatrists. More than $90 \%$ of psychiatrists did not offer depot treatment after a first psychotic episode. Psychiatrists tend to use depot antipsychotics in a conservative way, although they attribute positive outcomes to the method. Patients' negative attitudes might be related to the limited information they received about the medication. To enhance the use of depot antipsychotics, the information practices of psychiatrists should be improved, and patients should be informed about different forms of treatment during the early stages of their illness. ${ }^{14)}$

Non-adherence can be accurately measured and drug concentrations can be maintained in a stable state when depot medications are used. Therapists can prescribe the lowest effective dose as depot medications avoid first- 
pass metabolism and maintain a stable plasma concentration. ${ }^{15)}$ For these reasons, LAI was at first used mainly in chronic, stabilized patients for enhancing treatment medication adherence. Jaeger and Rossler ${ }^{14)}$ surveyed the attitudes of psychiatrists towards LAI and found that more than $90 \%$ of the responding psychiatrists never or rarely recommend changing to depot medication after a first episode. However, the recommendation rate increased to $50 \%$ after the experience of several relapses. There are several reasons to use LAI in chronic, stabilized schizophrenia patients including the delayed disappearance of medication-related adverse events among patients who experience adverse events such as akathisia and neuroleptic-induced extrapyramidal symptoms after the use of the initial LAI medications. There are limited numbers of medications available in the LAI formulation and it is not easy to adjust small doses. LAI treatment can also give patients a feeling of being controlled, and patients can be skeptical of the chemical treatment of mental symptoms. $^{15)}$

Of the disadvantages of using LAI, extrapyramidal adverse events are a major concern. LAI was mainly used for stable patients being switched from other medications. ${ }^{16)}$ However, second-generation antipsychotics (SGAs) are now rapidly replacing older conventional antipsychotics. ${ }^{17)}$ As SGAs have become clinically available, the risks of extrapyramidal adverse events have decreased. LAI formulations of SGAs have been introduced as another useful treatment option. Risperidone LAI was the first licensed SGA-LAI. Currently, paliperidone palmitate is also marketed in Korea and there are several new SGA-LAI compounds, including aripiprazole, that are under development. ${ }^{18)}$ Risperidone LAI (RLAI), an SGALAI, was evaluated in a non-randomized, open-label, 48-week study for schizophrenia patients who were symptomatically stable. Forty patients were enrolled, and 25 patients completed this study. The therapeutic response rate was $36.1 \%$ at the last observation carried forward and $48 \%$ by completer's analysis. Scores on clinical global impressions, the positive and negative syndrome scale (PANSS) subscales and total were significantly decreased over a 48-week period. ${ }^{19)}$ RLAI was also compared with oral antipsychotics over 24 months in another open-label study. The RLAI group included 50 patients and the oral risperidone or haloperidol group included 47 patients. The RLAI treatment group showed greater reduction of the PANSS total score, higher remission rates and a lower relapse rate among the responders, significantly fewer extrapyramidal symptoms, and significantly greater weight gain when compared with the oral antipsychotic agents (risperidone and haloperidol). ${ }^{20)}$

The indications of SGA-LAI have been extended from chronic, stabilized patients to acute psychotic patients as the risk for uncontrollable extrapyramidal motor adverse events has decreased. LAI was used in patients with an acute episode of schizophrenia during hospitalization. The Safety and Profile of Handling and Employing of Risperdal Consta in Emergency/Acute Care Settings (SPHERE) study was performed in a large cohort of patients with acute psychotic exacerbation. ${ }^{21)}$ This was an observational, non-interventional, multicenter, retrospective study with a large cohort of schizophrenia patients with acute psychotic symptoms in a real-world clinical setting. A total of 1,232 patients were enrolled in the study. All patients received RLAI and were discharged while maintaining RLAI treatment. Common adverse events were extrapyramidal symptoms $(1.1 \%)$, somnolence $(0.9 \%)$, tremor $(0.6 \%)$, dysarthria $(0.5 \%)$, akathisia $(0.4 \%)$, and impotence $(0.4 \%)$. This study showed that RLAI was well tolerated even in acute psychotic patients.

The use of LAI formulations is affected by various factors from the real world of clinical practice besides non-adherence, lack of efficacy, or adverse effects. LAIs are less frequently used in the United States than in Western Europe and Australia. ${ }^{22,23)}$ This difference can possibly be attributed to the differences in community mental health services and the health insurance systems.

\section{WHAT ABOUT USING LAI FORMULATIONS IN FIRST-EPISODE SCHIZOPHRENIA?}

It was reported that most clinical and psychosocial deterioration in schizophrenia occurs within the first 5 years from the onset of the illness. ${ }^{8)}$ Schizophrenia is thought to be a genetically-mediated neurodevelopmental disorder. The onset of the definite symptoms of schizophrenia is generally preceded by a prodromal phase. The functional deterioration process predominantly occurs during the pre-psychotic prodromal period and the first 5-10 years after the first episode. Accordingly, it is important to prevent relapses in the early stages of first-episode schizophrenia. ${ }^{9)}$ Although LAI were considered to be adherence-enhancing and highly preventive of relapse, the majority of psychiatrists seemed to avoid prescribing depot medication for patients with first-episode schizophrenia. Among the 246 psychiatrists attending the eighth World Congress of Biological Psychiatry, 71.1\% answered 'not using first-generation antipsychotics long acting injection 
(FGA-LAI) in first-episode patients' and 64.5\% indicated the same for SGA-LAI. ${ }^{24)}$ However, this belief was not supported by subsequent clinical studies.

There have been a few studies on the use of FGA-LAI in first-episode schizophrenia. One large prospective cohort study compared perphenazine depot with oral antipsychotics in patients hospitalized for the first time due to schizophrenia or schizoaffective disorder. ${ }^{25)}$ In a study of 2,230 hospitalized patients, perphenazine depot was associated with the lowest rates of discontinuation and lowest risk of rehospitalization when compared with oral haloperidol, olanzapine, clozapine, and risperidone over a mean follow-up period of 3.6 years. Kane et al ${ }^{26)}$ reported on 28 recently recovered first episode schizophrenia patients who were randomly assigned to oral fluphenazine hydrochloride, fluphenazine decanoate long acting injection, or placebo. Seven of the 17 patients receiving placebo experienced a relapse, whereas none of drug-treated patients experienced a relapse during study period of 1 year.

Research supporting the usefulness of SGA-LAI in first-episode schizophrenia has been mainly performed using RLAI since RLAI was the first marketed SGA-LAI. Emsley et al. ${ }^{27,28)}$ assessed the efficacy of RLAI $(25-50 \mathrm{mg}$ per every two weeks) among 179 patients who were diagnosed with cases of schizophreniform disorder or schizophrenia for two years in a single-site open-label study. In the study, 50 patients with first-episode schizophrenia were included in the analyses and 36 of these 50 patients (72\%) completed the trial, suggesting a relatively low discontinuation rate. ${ }^{27)} \mathrm{A}$ reduction of at least $50 \%$ on the PANSS total score was obtained by 42 of the 50 first-episode patients (84\%). ${ }^{27)}$ Thirty-two patients (64\%) met the Remission in Schizophrenia Working Group (RSWG) remission criteria and 31 patients (97\%) among that 32 maintained this remission status for two years until the completion of the study. ${ }^{28)} \mathrm{Kim}$ et $a l .{ }^{29)}$ also examined whether LAI could effectively act to prevent relapse in first-episode schizophrenia in a prospective, naturalistic, controlled, and open-label study conducted over 2 years in 50 patients with first-episode schizophrenia. Twenty-two patients with schizophrenia were assigned to the RLAI group and 28 patients with schizophrenia to the oral risperidone group as a control. They compared medication adherence, time to non-adherence, and relapse rate between the RLAI and control groups. The RLAI group showed a significantly lower relapse rate and higher rate of medication adherence than the control group. The results demonstrated that time to non-adherence was asso- ciated with the group difference and that RLAI could be effective even in first-episode schizophrenia patients.

Recently, Weiden et al. ${ }^{30)}$ conducted a prospective randomized controlled trial to compare acceptance and adherence between RLAI and continued oral atypical antipsychotics including risperidone, haloperidol, olanzapine, and quetiapine in first-episode schizophrenia patients. The study reported on only the early evaluation period and the first 12 weeks. Patients who were treated with RLAI (as-actually-treated analysis: 89\%, 95\% confidence interval, 64-97\%) were significantly more adherent than patients who were taking oral medications (as-actuallytreated analysis: 59\%, 95\% confidence interval, 32-78\%).

Bartzokis et al. ${ }^{31)}$ evaluated the treatment effects of RLAI on frontal lobe white matter volume in first-episode schizophrenia patients. Their randomized 6-month trial using inversion recovery magnetic resonance image image revealed that the frontal lobe white matter volume remained stable in the RLAI group whereas it significantly decreased in the oral risperidone group. This finding suggests that RLAI can improve the trajectory of myelination in first-episode patients and may be associated with positive effects on cognitive functions.

Additionally, first-episode schizophrenia patients are generally younger than chronic schizophrenia patients. Using LAIs in first episode schizophrenia may achieve more predictable and stable plasma concentrations of antipsychotic drug compared with chronic schizophrenia patients due to age-related changes affecting the pharmacokinetics of antipsychotic drugs. ${ }^{32)}$ Thus, it seems to be relatively easy to prescribe LAIs for first-episode schizophrenia to load appropriate doses of drug and minimize the risk for adverse effects and drug interaction. ${ }^{33)}$

Using LAI in first-episode schizophrenia may have beneficial impacts on treatment outcomes. Especially in terms of adherence and relapse prevention, prescribing SGA-LAI might be another treatment option even in active psychotic first-episode schizophrenia. However, there is limited research data (mainly focused on RLAI) for clinical trials in first-episode schizophrenia. We need more well-designed, randomized controlled clinical trials for the use of SGA-LAIs in first-episode schizophrenia.

\section{CONCLUSIONS}

In terms of adherence, using LAI for first-episode schizophrenia is cautiously proposed as a possible treatment option. Because first-episode schizophrenia patients frequently experience relapses during the first years of 
their illness and because relapses are related with functional deterioration and poor treatment results, successful management of first-episode schizophrenia is important for better long-term outcomes. However, supporting evidence for the use of LAI in first-episode schizophrenia is not enough, and research on LAI has been conducted mainly with RLAI. Studies about the adverse effects of SGA-LAI in long term use such as tardive dyskinesia or metabolic effects are also needed. Additionally, multilateral efforts to solve problems in mental health services, health insurance, and ethical issues related to prescribing LAIs for first-episode schizophrenia patients are required prior to considering LAIs as a future therapeutic option.

\section{REFERENCES}

1. Perkins DO. Predictors of noncompliance in patients with schizophrenia. J Clin Psychiatry 2002;63:1121-1128.

2. Yang J, Ko YH, Paik JW, Lee MS, Han C, Joe SH, et al. Symptom severity and attitudes toward medication: impacts on adherence in outpatients with schizophrenia. Schizophr Res 2012;134:226-231.

3. Glick ID, Stekoll AH, Hays S. The role of the family and improvement in treatment maintenance, adherence, and outcome for schizophrenia. J Clin Psychopharmacol 2011;31: 82-85.

4. Nasrallah HA. The case for long-acting antipsychotic agents in the post-CATIE era. Acta Psychiatr Scand 2007;115:260267.

5. Masand PS. A review of pharmacologic strategies for switching to atypical antipsychotics. Prim Care Companion $J$ Clin Psychiatry 2005;7:121-129.

6. Kane JM. Utilization of long-acting antipsychotic medication in patient care. CNS Spectr 2006;11(12 Suppl 14):1-7.

7. Birchwood M, Todd P, Jackson C. Early intervention in psychosis. The critical period hypothesis. Br J Psychiatry Suppl 1998; 172:53-59.

8. Lieberman J, Jody D, Geisler S, Alvir J, Loebel A, Szymanski S, et al. Time course and biologic correlates of treatment response in first-episode schizophrenia. Arch Gen Psychiatry 1993;50:369-376.

9. Lieberman JA, Perkins D, Belger A, Chakos M, Jarskog F, Boteva $\mathrm{K}$, et al. The early stages of schizophrenia: speculations on pathogenesis, pathophysiology, and therapeutic approaches. Biol Psychiatry 2001;50:884-897.

10. Robinson D, Woerner MG, Alvir JM, Bilder R, Goldman R, Geisler S, et al. Predictors of relapse following response from a first episode of schizophrenia or schizoaffective disorder. Arch Gen Psychiatry 1999;56:241-247.

11. Carpenter WT Jr, Heinrichs DW, Hanlon TE. A comparative trial of pharmacologic strategies in schizophrenia. Am J Psychiatry 1987;144:1466-1470.

12. Herz MI, Glazer WM, Mostert MA, Sheard MA, Szymanski $\mathrm{HV}$, Hafez $\mathrm{H}$, et al. Intermittent vs maintenance medication in schizophrenia. Two-year results. Arch Gen Psychiatry 1991;48:333-339.

13. Gaebel W, Riesbeck M, Wölwer W, Klimke A, Eickhoff M, von Wilmsdorff M, et al.; German Study Group on FirstEpisode Schizophrenia. Relapse prevention in first-episode schizophrenia-maintenance vs intermittent drug treatment with prodrome-based early intervention: results of a rando- mized controlled trial within the German Research Network on Schizophrenia. J Clin Psychiatry 2011;72:205-218.

14. Jaeger M, Rossler W. Attitudes towards long-acting depot antipsychotics: a survey of patients, relatives and psychiatrists. Psychiatry Res 2010;175:58-62.

15. Gerlach J. Depot neuroleptics in relapse prevention: advantages and disadvantages. Int Clin Psychopharmacol 1995; 9(Suppl 5):17-20.

16. Taylor DM, Young CL, Mace S, Patel MX. Early clinical experience with risperidone long-acting injection: a prospective, 6-month follow-up of 100 patients. J Clin Psychiatry 2004;65:1076-1083.

17. Geddes J. Generating evidence to inform policy and practice: the example of the second generation "atypical" antipsychotics. Schizophr Bull 2003;29:105-114.

18. Agid O, Foussias G, Remington G. Long-acting injectable antipsychotics in the treatment of schizophrenia: their role in relapse prevention. Expert Opin Pharmacother 2010;11: 2301-2317.

19. Lee MS, Ko YH, Lee SH, Seo YJ, Kim SH, Joe SH, et al. Long-term treatment with long-acting risperidone in Korean patients with schizophrenia. Hum Psychopharmacol 2006; 21:399-407.

20. Emsley R, Oosthuizen P, Koen L, Niehaus DJ, Medori R, Rabinowitz J. Oral versus injectable antipsychotic treatment in early psychosis: post hoc comparison of two studies. Clin Ther 2008;30:2378-2386.

21. De la Gándara J, San Molina L, Rubio G, Rodriguez-Morales A, Hidalgo Borrajo R, Burón JA. Experience with injectable long-acting risperidone in long-term therapy after an acute episode of schizophrenia: the SPHERE Study. Expert Rev Neurother 2009:9:1463-1474.

22. Shi L, Ascher-Svanum H, Zhu B, Faries D, Montgomery W, Marder SR. Characteristics and use patterns of patients taking first-generation depot antipsychotics or oral antipsychotics for schizophrenia. Psychiatr Serv 2007;58:482-488.

23. Mond J, Morice R, Owen C, Korten A. Use of antipsychotic medications in Australia between July 1995 and December 2001. Aust N Z J Psychiatry 2003;37:55-61.

24. Heres S, Hamann J, Kissling W, Leucht S. Attitudes of psychiatrists toward antipsychotic depot medication. J Clin Psychiatry 2006;67:1948-1953.

25. Tiihonen J, Wahlbeck K, Lönnqvist J, Klaukka T, Ioannidis JP, Volavka J, et al. Effectiveness of antipsychotic treatments in a nationwide cohort of patients in community care after first hospitalisation due to schizophrenia and schizoaffective disorder: observational follow-up study. BMJ 2006;333:224.

26. Kane JM, Rifkin A, Quitkin F, Nayak D, Ramos-Lorenzi J. Fluphenazine vs placebo in patients with remitted, acute first-episode schizophrenia. Arch Gen Psychiatry 1982;39: 70-73.

27. Emsley R, Medori R, Koen L, Oosthuizen PP, Niehaus DJ, Rabinowitz J. Long-acting injectable risperidone in the treatment of subjects with recent-onset psychosis: a preliminary study. J Clin Psychopharmacol 2008;28:210-213.

28. Emsley R, Oosthuizen P, Koen L, Niehaus DJ, Medori R, Rabinowitz J. Remission in patients with first-episode schizophrenia receiving assured antipsychotic medication: a study with risperidone long-acting injection. Int Clin Psychopharmacol 2008;23:325-331.

29. Kim B, Lee SH, Choi TK, Suh S, Kim YW, Lee E, et al. Effectiveness of risperidone long-acting injection in first -episode schizophrenia: in naturalistic setting. Prog Neuropsychopharmacol Biol Psychiatry 2008;32:1231-1235.

30. Weiden PJ, Schooler NR, Weedon JC, Elmouchtari A, Suna- 
kawa A, Goldfinger SM. A randomized controlled trial of long-acting injectable risperidone vs continuation on oral atypical antipsychotics for first-episode schizophrenia patients: initial adherence outcome. J Clin Psychiatry 2009;70: 1397-1406.

31. Bartzokis G, Lu PH, Amar CP, Raven EP, Detore NR, Altshuler LL, et al. Long acting injection versus oral risperidone in first-episode schizophrenia: differential impact on white matter myelination trajectory. Schizophr Res 2011;132: 35-41.

32. Masand PS, Gupta S. Long-acting injectable antipsychotics in the elderly: guidelines for effective use. Drugs Aging 2003;20:1099-1110.

33. Masand PS. Side effects of antipsychotics in the elderly. $J$ Clin Psychiatry 2000;61(Suppl 8):43-49. 